\title{
A review of developments in the management of retinal diseases
}

\author{
Somnath Banerjee
}

J R Soc Med 2006;99:125-127

\section{INTRODUCTION}

Retinal disease continues to be a major contributor to poor vision especially in the ageing population. Age related macular degeneration is the main cause of registerable blindness in the developed world. Retinal vein occlusions are the second most common retinal vascular disease following diabetic retinopathy.

This article is a review of new advances in the management of these conditions as well as an introduction to developments in the surgical management of retinal disease using new technology.

\section{METHODS}

A review of the ophthalmic literature as well as material obtained from presentations at national and international conferences was used to provide the material in this article.

\section{MANAGEMENT OF AGE RELATED MACULAR DEGENERATION}

Age-related macular degeneration is the most common cause of poor vision in those over 65 in the Western world. There are two types: dry (atrophic) age-related macular degeneration which accounts for $85-90 \%$ of all cases but is associated with a better prognosis; and wet (exudative) agerelated macular degeneration (Figure 1). Wet age-related macular degeneration is associated with choroidal neovascularization, a process similar to angiogenesis at other sites.

The benefit of thermal (argon) laser retinal photocoagulation was established for choroidal neovascular membranes away from the fovea (extrafoveal) in the Macular Photocoagulation Study in the early 1990s. ${ }^{1}$ In photodynamic therapy ${ }^{2,3}$ a light-sensitive agent (Verteporfin) is given by intravenous infusion over $10 \mathrm{~min}$ at a dose of $6 \mathrm{mg} / \mathrm{m}^{2}$ of body surface area. Fifteen minutes after the start of the infusion, a low-powered laser calibrated to deliver a set amount of energy at a specific wavelength is applied over a circular area slightly larger than the choroidal neovascular membranes. The laser is not powerful enough to cause any damage on its own but activation of

Department of Ophthalmology, Leicester Royal Infirmary, Infirmary Road, Leicester LE1 5WW, UK

E-mail: pinkubanerjee2002@yahoo.co.uk
Verteporfin by light absorption results in formation of cytotoxic free radicals that damage the new blood vessels of the choroidal neovascular membranes. photodynamic therapy has been shown to reduce the rate of visual loss in patients with well-defined (classic) choroidal neovascular membranes but this accounts for no greater than $20 \%$ of patients with wet age-related macular degeneration. Recent studies have shown that photodynamic therapy may be beneficial for poorly defined (occult) choroidal neovascular membranes. Medicaid recently agreed to reimburse practitioners for photodynamic therapy for occult choroidal neovascular membranes in the USA. If it is advocated also by the FDA, a very large number of patients will be eligible with considerable impact on manpower and resources. In the UK, NICE has advocated photodynamic therapy only for predominantly classic choroidal neovascular membranes at present. This guidance will due for review in 2006-2007

Anecortave acetate is an angiostatic agent administered by posterior juxtascleral injection close to the macula every 6 months. A recent 24-months double-masked phase 2/3 study showed that it was superior to placebo in stabilizing vision for all types of choroidal neovascular membranes. The Anecortave Acetate Risk Reduction Trial (AARRT) is examining whether anecortave reduces progression of dry age-related macular degeneration in patients at high risk of progression to wet age-related macular degeneration. Macugen is a highly selective inhibitor of vascular endothelial growth factor with anti-angiogenic and antipermeability effects. The vascular endothelial growth factor Inhibition Study in Ocular Neovascularisation (VISION) demonstrated improved visual outcome at 54 weeks in wet age-related macular degeneration, regardless of lesion subtype. Macugen has to be delivered by intra-vitreal injection every 6 weeks. Lucentis is a recombinant humanized antibody fragment that binds to vascular endothelial growth factor and inactivates all vascular endothelial growth factor isoforms. This is also delivered by intravitreal injection on a monthly basis.

\section{MANAGEMENT OF RETINAL VEIN OCCLUSION}

Central or branch retinal vein occlusion is the second most common retinal vascular disease after diabetic retinopathy (Figure 2) Current practise is to follow the guidelines 


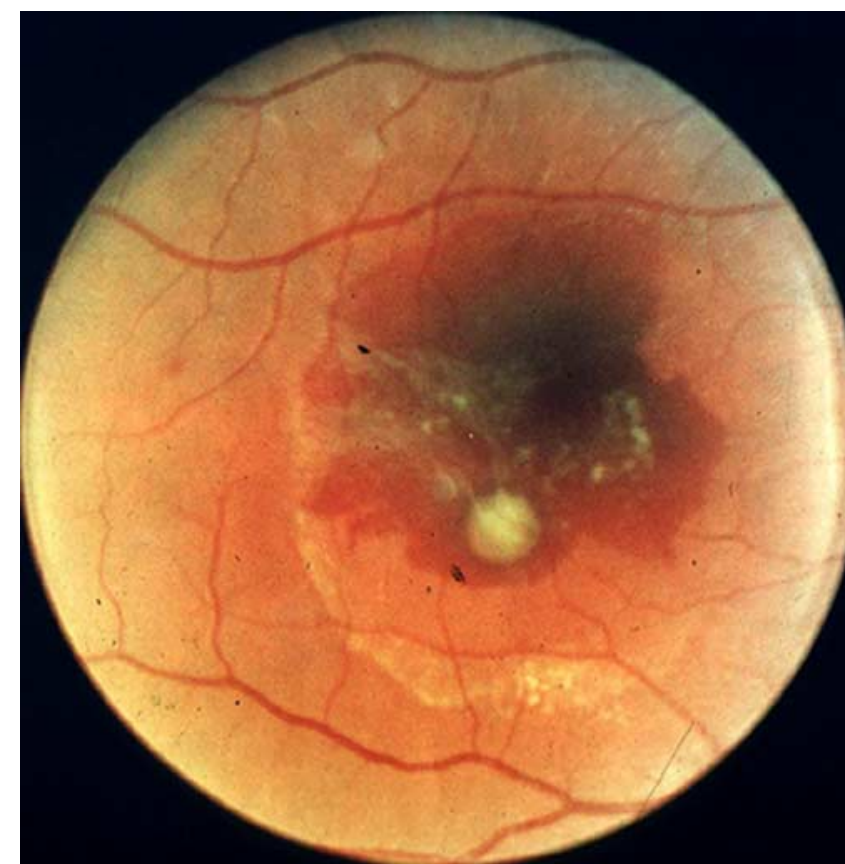

Figure 1 Wet AMD with macular haemorrhage (in colour online)

suggested by the Central Retinal Vein Occlusion Study ${ }^{4}$ and the Branch Retinal Vein Occlusion Study. ${ }^{5}$ The management of central retinal vein occlusion (central retinal vein occlusion) involves identifying modifiable risk factors, such as systemic hypertension and hyperlipidaemia, and treating ischaemic complications of the vascular occlusion. Laser treatment (pan retinal photocoagulation) is indicated at the earliest sign of iris neovascularization. Macular laser photocoagulation is not beneficial to visual improvement although the oedema may regress. It is therefore not recommended in central retinal vein occlusion. In branch retinal vein occlusion a modified grid laser photocoagulation is indicated for macular oedema persisting for three or more months and the visual acuity is $6 / 12$ or less in the absence of significant macular ischaemia.

There are a number of other suggested techniques to treat retinal vein occlusions, including creating an anastomosis between the retina and choroid using high energy argon laser. ${ }^{6}$ Surgically incising the nasal part of the optic nerve (radial optic neurotomy) is based on the theory that the site of occlusion in central retinal vein occlusion is in the substance of the optic nerve producing a 'compartment syndrome'. ${ }^{7}$ A randomized study is currently taking place (Radial Optic Neurotomy in Central Vein Occlusion Study sponsored by NIH). If an occlusion site can be identified in branch retinal vein occlusion, surgical incision of the common adventitial sheath around the retinal arteriole and vein (arterio-venous sheathotomy) with

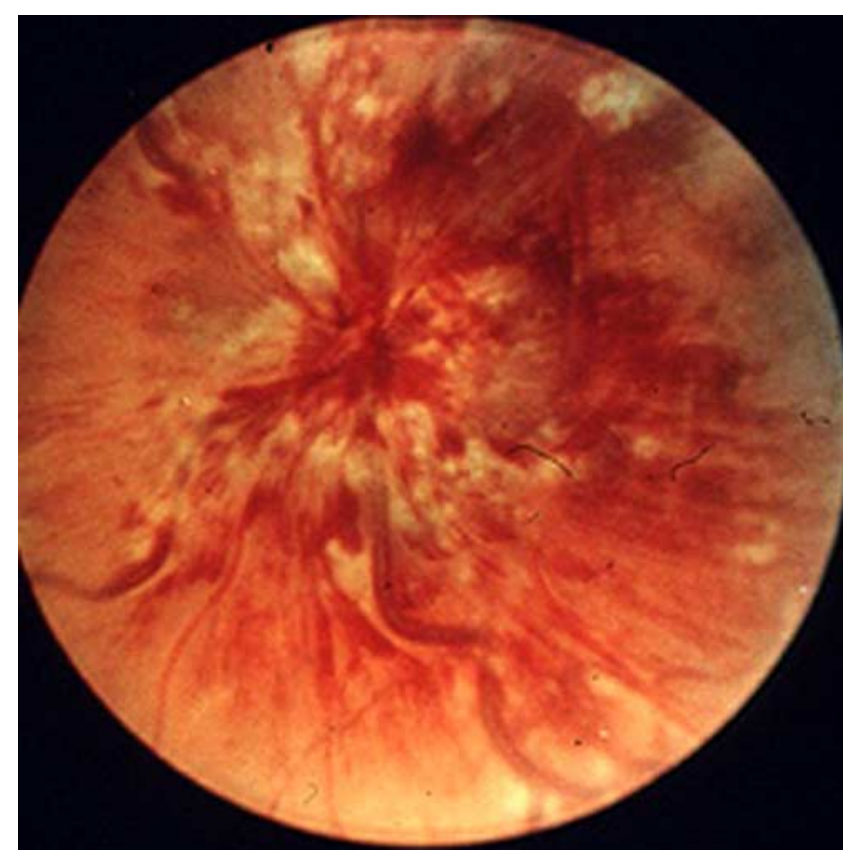

Figure 2 Central retinal vein occlusion (in colour online)

because it is thought that the vein is compressed by the atherosclerotic artery. The procedure now most commonly performed for macular oedema following vein occlusion is the injection of intravitreal steroid (triamcinolone). ${ }^{8}$ However, this procedure maybe associated with increased intraocular pressure and does not always result in visual improvement. Randomised controlled trials on the efficacy of intravitreal triamcinolone in the management of retinal vein occlusion are awaited.

\section{SURGICAL ADVANCES-TRANSCONJUNCTIVAL SUTURELESS VITRECTOMY}

Removal of the vitreous using an aspiration-cutting instrument inserted through the pars plana (pars plana vitrectomy) is performed for a number of conditions including retinal detachment, complications of diabetic retinopathy and certain macular pathology. Machemer first performed pars plana vitrectomy in the early 1970's using a 17 -gauge $(1.5 \mathrm{~mm}$ diameter) instrument. This required a $2.3 \mathrm{~mm}$ scleral incision. In 1974 O'Malley designed a smaller 20-gauge $(0.9 \mathrm{~mm})$ instrument, which is currently in widespread use. Since 1990, De Juan et al. have been developing a 25 gauge $(0.5 \mathrm{~mm})$ system. ${ }^{9}$ More recently, Eckhardt has modified this to a 23-gauge system. The advantages of both these systems are faster entry and closure, as no suturing of entry sites is required (transconjunctival sutureless vitrectomy), and quicker 
recovery for the patient. Both are available in this country and are likely to be used more widely with time.

\section{CONCLUSION}

This is an exciting time for the retinal specialist with treatment options now available for conditions that were previously not amenable to any type of therapy.

As with any new developments in medicine it is wise to err on the side of caution when advising patients as to the potential benefits of these until long-term outcomes become available.

As sutureless vitrectomy goes, this procedure which used to be associated with long operating times and stay in hospital as well as recovery times this may soon also be a thing of the past.

\section{REFERENCES}

1 Macular Photocoagulation Study Group. Laser photocoagulation of subfoveal neovascular lesions of age related macular degeneration. Updated findings from two clinical trials. Arch Ophthalmol 1993;111:1200-9

2 Verteporfin In Photodynamic Therapy Study Group. Verteporfin therapy of subfoveal choroidal neovascularisation in age-related macular degeneration: two-year results of a randomized clinical trial including lesions with occult with no classic choroidal neovascularisation. Am J Ophthalmol 2001;131:541-60

3 Blumenkranz MS, Bressler NM, Bressler SB, el al. Verteporfin therapy for subfoveal choroidal neovascularisation in age-related macular degeneration: three-year results of an open-label extension of 2 randomised clinical trials - TAP Report No 5. Arch Ophthalmol 2002; 120:1307-13

4 The Central Vein Occlusion Study Group. Natural history and clinical management of central retinal vein occlusion. Arch Ophthalmol 1997; 115:486-91

5 The Branch Vein Occlusion Study Group. Argon laser photocoagulation for macular edema in branch vein occlusion. Am J Ophthalmol 1984; 98:271-82

6 McAllister IL, Douglas JP, Constable IJ, et al. Laser induced chorioretinal venous anastomosis for nonischemic central retinal vein occlusion: evaluation of the complications and their risk factors. Am J Ophthalmol 1998;126:219-29

7 Opremcak EM, Bruce RA, Lomeo MD, et al. Radial optic neurotomy for central retinal vein occlusion: a retrospective pilot study of 11 consecutive patients. Retina 2001;21:408-15

8 Jonas JB, Kreissig I, Degenring RF. Intravitreal triamcinolone acetonide as treatment of macular edema in central retinal vein occlusion. Graefes Arch Clin Exp Ophthalmol 2002;240;782-3

9 Fujii GY, De Juan E Jr, Humayun MS, et al. Initial experience using the transconjunctival sutureless vitrectomy system for vitreoretinal surgery. Ophthalmology 2002;109:1814-20 